\title{
Brain-Computer Interface-Progress and Prospects
}

\author{
Yuchen $\mathrm{Wu}^{1}$ \\ ${ }^{1}$ Lakefield College School, K0L2H0, Lakefield, Ontario, Canada
}

\begin{abstract}
Since the advent of Brain-Computer Interface (BCI), this technology has been significantly contributed modern society in many aspects such as medical and informational science. With further approaches in this interdisciplinary technology and based on current research, BCI is considered to be the potential solution to medical or surgical difficulties such as restoration of neurological function or motor abilities. In this article, the current state of BCI development in multiple platforms was briefly introduced. By organizing and analyzing laboratory data from the state-of-the-art BCI research, this article also illustrated the breakthrough on different BCI systems based on the lab data. The multitude of applications and contributions in medical science and engineering of both invasive and non-invasive systems were also discussed with the help of clinical data. Eventually, the potential and future attempts will be projected and inferred based on the present state of such connection in this article. After comparing and contrasting two types of interfaces and analysis, a conclusion could be made that invasive systems will eventually surpass noninvasive methods in more applications areas due to its preponderance of precise control.
\end{abstract}

\section{Introduction}

A brain-computer interface $(\mathrm{BCI})$, also called a neural control interface (NCI), mind-machine interface (MMI), direct neural interface (DNI), or brain-machine interface (BMI), is usually referring to a direct communication pathway which receives and conducts bio signals to proceed artificial output [1]. In this way, BCI connects biological tissues directly to an external device to restore original function or achieve enhancement. Depending on the method of connections, BCI platforms can be generally divided into two systems with distinct pros and cons [2]:

Invasive Systems: Data receiving and processing devices are implanted directly into the grey matter of brain hence quality and precision of signals collected through this method are usually higher

Non-Invasive Systems: Devices are placed externally to the brain, or other method are applied to receive bio signals such as EEG. Usually easier to adapt and show less clinical risks.

Despite the explosive breakthrough in information and material technology in the 21 st century have permitted approaches in brain-computer integration, the development and clinical application of BCI is still limited due to limitations in data collection, transport, and clinical risks. Although many of the BCI platforms are in their premature phrase for advanced application such as a full neural restoration or commercial use, a number of them are still applied to help patients in scale. For example, the artificial cochlear technology has been one of the most success early BCI application where electric signals are transduced to the brain through artificial devices.
In order to acquire higher data precision for higherlevel data transmission, companies such as Neuralink are devoted in improving invasive interfaces while minimizing potential clinical risks. By closely analyzing information and data on the current improvement in BCI platforms, this article would focus on how these attempts permit higher data transmission rate and precision. Specifically, some of the cut-edge technology development of distinct interfaces will be analyzed, along with projections on future $\mathrm{BCI}$ development and clinical or commercial applications based on the current studies. The rapid advancement of brain-computer interfaces gives us an insight of potential solutions in technical difficulties in many aspects. Through this article, this interdisciplinary research could be more accessible to the public and driven more attention in discussion and research.

\section{Current Development of BCI Platform}

\subsection{Current Approach in Invasive Systems and Surgical Improvement}

In order to acquire higher data precision to optimize utilization of data and carry better function, companies such as Neuralink have been devoted in improving data transmission of invasive BCI systems in several ways. On July 17, 2019, Elon Musk published a new research achievement on BCI interface and proclaimed its potential in commercial use.

Compared with previous approaches in invasive interfaces, Neuralink used fine, flexible multi-electrode polymer neural probes made of polyimide to embrace bio-

\footnotetext{
* Corresponding author: ShiLiShuang@cas-harbour.org
} 
compatibility [3]. All of the implanted threads have typical thickness of 4 to $6 \mu \mathrm{m}$. The thread areas together with sensor areas which are connected to chips that collect and amplifies signals forms the basic unit of a thin film array. Each array normally contains 48 to 96 arrays, each of them also contains 32 independent electrodes. The thread and electrode type also vary, such as the Linear Edge" probes, with 32 electrode contacts spaced by $50 \mu \mathrm{m}$, and the "Tree" probes with 32 electrode contacts spaced by $75 \mu \mathrm{m}$ to fit all situations. Together, group of 10 thin film devices are patterned on a wafer each with electrodes. This allows higher data transduction capacity compared with previous 128 electrodes from BrainGate [4]. These thread transfer electrodes to specific areas to the brain which allow precise recording of single action potentials in scale.

Neuralink also developed a robotic insertion method to implant flexible probes [3]. The neurosurgery robot performs implantation promised precision and minimizes deviation compared with a surgical operation. It also avoids blood vessel to the highest extent, lower the possibility of potential inflammation, rejection and other side reactions. The robot's inserter head contains a needle and a pincher which cooperate to stabilize and insert each electrode individually like a sewing machine. The needle has a diameter of $24 \mu \mathrm{m}$, driven by a linear motor which make insertion and retraction speed controllable. The pincher is a tungsten wire that is $50 \mu \mathrm{m}$ in length with high operability. The robot commits $10 \mu \mathrm{m}$ globally accurate, which minimizes damage to biological tissues. The inserter head also includes a range of optical devices, such as brain position sensor; light modules and stereoscopic cameras of different angles to assist orientation and highly precise operation.
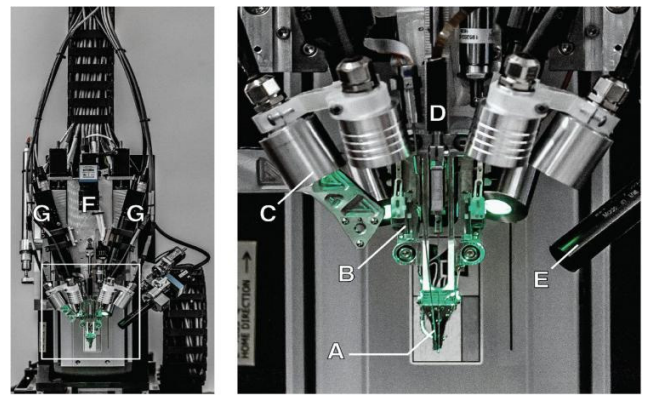

Fig. 1. The robotic electrode inserter; enlarged view of the inserter-head shown in the inset. A. Loaded needle pincher cartridge. B. Low-force contact brain position sensor. C. Light modules with multiple independent wavelengths. D. Needle motor. E. One of four cameras focused on the needle during insertion. F. Camera with wide angle view of surgical field. G. Stereoscopic cameras [27].

The team also upgrade electronics to receive, record, analyze and amplify signals from thousands of electrodes chronically with minimal power rate and size. Built on Neuralink's custom application specific integrated circuit (ASIC), each neural processing electronic consists 256 channels of data that are individually programmable and with low power consumption. Together the packaged device holds up to 3072 channels which maximizes the efficiency of real time processing. The design ASIC also integrates on-chip analog-to-digital converters (ADCs)
$19.3 \mathrm{kHz}$ with 10 bit resolution, and peripheral control. Musk's team also specify two output channel recording systems that connects ASIC as the essential recording platform and an ethernet-connected base station that provides downstream users with access to the recorded data.

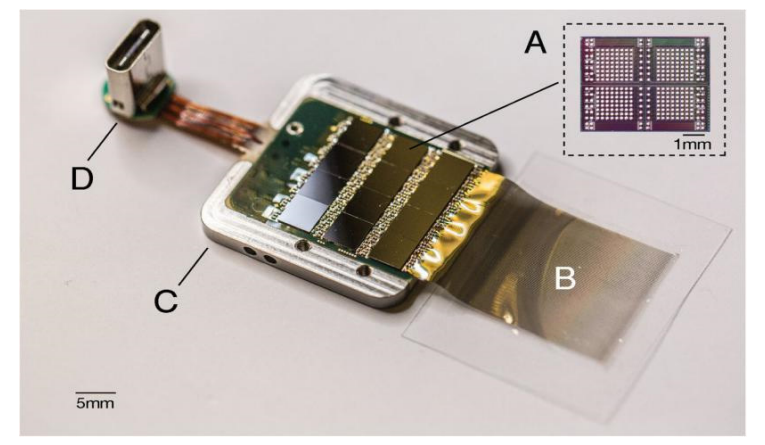

Fig. 2. A packaged sensor device. A. individual neural processing ASIC capable of processing 256 channels of data. This particular packaged device contains 12 of these chips for a total of 3,072 channels. B. Polymer threads on parylene-c substrate. C. Titanium enclosure (lid removed). D. Digital USB-C connector for power and data [27].

\subsection{Non-Invasive Systems}

\subsubsection{EEG}

Electroencephalogram (EEG) indicates the integration of all electric signal of cortical neurons. It is a method of recording the simultaneous postsynaptic potential changes of neurons during cerebral activities, which outputs the overall reflection of the electrophysiological activities of brain nerve cells on the cerebral cortex or scalp surface [4]. Generally, multiple EEG electrode pads are closely attached to different positions of the user's scalp to collect spontaneous and rhythmic brain electrical signals. The back end is connected to a single-channel or multi-channel circuit system, and exports corresponding graphics by amplifying the eclectic signal cascades [5]. Through EEG, electric signals of specific cerebral functional areas could be collected respectively for analyzing corresponding functional states. Brain waves could be divided into five frequency bands (Delta: $1-3 \mathrm{~Hz}$, Theta: 4-7HZ, Alpha: 8-13Hz, Beta: 14-30Hz, Gamma: $>30 \mathrm{~Hz}$ ) based on the corresponding rhythmic physiological activities of the brain [6].

Table. 1. EEG Frequency Bands and Its Corresponding Brain State [28].

\begin{tabular}{|c|c|c|}
\hline Brainwave Type & $\begin{array}{l}\text { Frequency Range } \\
(\mathrm{Hz})\end{array}$ & State of the brain \\
\hline Delta ( $(\tilde{)})$ & $0.1 \mathrm{~Hz}$ to $3 \mathrm{~Hz}$ & $\begin{array}{l}\text { Deep, dreamless sleep, non-REM sleep, } \\
\text { unconscious }\end{array}$ \\
\hline Theta ( $(\theta)$ & $4 \mathrm{~Hz}$ to $7 \mathrm{~Hz}$ & $\begin{array}{l}\text { Intuitive, creative, recall, fantasy, imaginary, } \\
\text { dream }\end{array}$ \\
\hline Alpha $(\alpha)$ & $8 \mathrm{~Hz}+\mathrm{l} 12 \mathrm{~Hz}$ & Relaxed, but not drowsy, tranquil, conscious \\
\hline Low-range Beta ( $\beta$ ) & $12 \mathrm{~Hz}$ to $15 \mathrm{~Hz}$ & Formerly SMR, relaxed yet focused, integrated \\
\hline Mid-range Beta ( $\beta$ ) & $16 \mathrm{~Hz}$ to $20 \mathrm{~Hz}$ & Thinking, aware of self \& surroundings \\
\hline High-range Beta ( $\beta$ ) & $21 \mathrm{~Hz}$ to $30 \mathrm{~Hz}$ & Alertness, agitation \\
\hline Gamma $(\gamma)$ & $30 \mathrm{~Hz}$ to $100, \mathrm{~Hz}$ & Motor Functions, higher mental activity \\
\hline
\end{tabular}


By monitoring the distinct rhythms located over specific brain areas, a direct pathway that connects human brain and external environmental could be created surpassing the regular physical signal transduction pathway [7]. Based on this hypothesis, EEG is commonly used in obtaining electric signals for signal exchange in non-invasive BCI interfaces. The signal collected by the non-invasive electrode is relatively weak and contains higher portion of noises, but due to its safety, convenience in operation and low cost, it is more common in application fields than invasive platforms which promises higher precision of signals. On the other hand, the problem of signal precision could be alleviated by improving the technique of sensor, circuit and algorithm that amplified obtained signals and filter noises. This is also the research focus on realization of BCI based on EEG interfaces.

A large class of EEG-based BCI systems relies on the detected BCI paradigms monitored by external EEG monitors and then interprete and translate to commands that control certain tasks. Common BCI paradigms, or common brains patterns that could be capture by EEG include Event-Related Desynchronization or Synchronization (ERD/ERS), Event Related Potentials (ERP) and Steady State Evoked Potentials (SSEP). The rhythmic electrophysiological phenomenon of increased or decresed electrical activities of cerebral cortex stimulated by sensory organs or subjective action commands are called ERD and ERS. The initiation of ERD and ERS requires no external stimulation, and they are fully controlled by the patients intention in initiating operation tasks and is self-controllable, however, such flexibility reduces the precision of signal processing, BCI systems requires increasing amount of practice from the users to output willing signals of specific tasks, and for the processing port, the precision decreases with increasing tasks [9]. By comparison, ERP generates when nueral systems underwent unique stimulations such as light and sound. The most representative ERP P300 is considered related with cognitive capacity of humans. ERP are natural physiological reaction which requires no previous training for users, and it occurs almost simultaneously with the stimulation which is beneficial for real-time analyse and control from the BCI end, but their amplitude are relatively lower and hard to detect, the deviation of ERP generation on an individual and specific external stimulation also brought technical difficulties. SSEP is the potential response recorded through scalp electrodes, it is stimulated by multiple continues, steadystate stimulus signals such as regular flashing lights and changing sounds. The strength of SSEP is directly related with user's focus on the stimulation, which also means thy could operate more tasks by changing attention. However, this method again requires continues stimulation and requires high focus over time from the users.

\subsubsection{VEP}

Visually evoked potentials are another type of brain physiological modulation that occur in the visual cortex of human brains after receiving visual stimulus [7]. Based on the frequency of simulations, VEP can be divided into two categories: transient VEPs (TVEPs) and as steadystate VEPs (SSVEPs). TVEPs occurs with visual simulations that are usually below $6 \mathrm{~Hz}$ while SSVEPs requires visual stimuli of higher frequencies. Any changes in the visual field could elicit TVEPs, however, SSVEP is more commonly used in BCI because they have identical frequency of the blinking frequency of the stimulus and are less susceptible to manual activities such as blinking and eye movements, or to electromyographic noise contamination. [10] BCI that based on SSVEPs allows users to artificially select the target with eyesight by visually focusing on the target object and BCI collected the data and identify the target with SSVEP features analysis. SSVEPs are the most widely used in steady-state evoked potentials based BCI systems for movement assistance or function restore, such as SSVEP-based wheelchair control and spelling control. [8] SSVEPs could be further classified into three categories based on the stimulus sequence modulation: time modulated VEP (t-VEP) BCIs, frequency modulated VEP (f-VEP) BCIs, and pseudorandom code modulated VEP (c-VEP) BCIs. Although c-VEP requires user training to obtain full control, it's still more suitable for application compared with other two methods due to its high data transfer rate, an online system target identification could reach an average an average information transfer rate of $108 \pm 12$ bits min -1 and a maximum of 123 bits min -1 for an individual object [11].

Due to the advantages of SSVEP such as high signal to noise ratio and information transfer rate, SSVEP-based $\mathrm{BCI}$ technology has become the research focus of many practical BCI applications. Generally development on such systems includes visual stimulus patterns, signal analysis and algorithm and signal collection side [12]. Ever since SSVEP-based BCI has been proven feasible in 2000 by the US ARir Force [13], many aspects of this platform has been improved with practical uses. Research from Xi'an Jiaotong University has studied the multitude of visual stimulus patterns and applied in wheelchain guiding system [14]. Research from Tsinghua University published the methods of SSVEP signal identifications and has successfully developed BCI system that assists dialing [12]. All of these researches improves the efficiency of signal communication and identification accuracy of BCI, as well as realized more functional applications. But SSVEP-based BCI need to overcome more technical difficulties to be used on higher functional applications. Information transfer rate, classification accuracy, noise resistance are all key checkpoints of the widespread application of SSVEP-based BCI platforms. Research from Tianjing University of Technology introduced Emotive EPOC to collect signals and process high accuracy offline analysis to obtain partial control of an artificial NAO robot [12]. Another research from Korea applied LED connected SSVEP system on typing and optimized the factors that have influence on the performance of the mental typing system, for example the distances between adjacent keys, light source arrangements, stimulating frequencies, layout of the electrodes, and visual angles, which has a result of 9.39 
letters per minute among five volunteers and average accuracy of $87.58 \%$ [15].

Table. 2. Classification accuracy for each participant with respectto different visual angles and electrode combinations. The highest classification accuracy of each participantis printed in bold [15]

\begin{tabular}{|c|c|c|c|c|c|c|c|c|}
\hline \multirow[t]{2}{*}{ Subjects } & \multirow[t]{2}{*}{ Visual angle } & \multicolumn{7}{|c|}{ Classification accuracy (\%) } \\
\hline & & $0 z$ & 01 & 02 & $0 z, 01$ & $0 z, 02$ & 01,02 & $0 z, 01,02$ \\
\hline \multirow[t]{2}{*}{ P1 } & $30^{\circ}$ & 91.66 & 81.66 & 73.33 & 85 & 88.33 & 85 & 86.67 \\
\hline & $40^{\circ}$ & 81.66 & 58.33 & 55 & 73.33 & 75 & 63.66 & 71.67 \\
\hline \multirow[t]{2}{*}{ P2 } & $30^{\circ}$ & 31.67 & 18.33 & 63.33 & 25 & 46.66 & 46.66 & 40 \\
\hline & $40^{\circ}$ & 26.66 & 13.33 & 56.67 & 21.66 & 43.33 & 41.67 & 36.67 \\
\hline \multirow[t]{2}{*}{ P3 } & $30^{\circ}$ & 15 & 33.33 & 71.67 & 23.33 & 41.67 & 53.33 & 41.67 \\
\hline & $40^{\circ}$ & 16.67 & 23.33 & 71.67 & 20 & 55 & 53.33 & 33.67 \\
\hline \multirow[t]{2}{*}{ P4 } & $30^{\circ}$ & 70 & 63.34 & 50 & 73.34 & 68.34 & 66.67 & 73.34 \\
\hline & $40^{\circ}$ & 63.34 & 56.67 & 48.34 & 65 & 65 & 60 & 65 \\
\hline \multirow[t]{2}{*}{ P5 } & $30^{\circ}$ & 80 & 65 & 76.67 & 75 & 83.34 & 75 & 75 \\
\hline & $40^{\circ}$ & 83.34 & 76.67 & 86.67 & 80 & 86.67 & 83.33 & 85 \\
\hline
\end{tabular}

\subsection{Application}

Today's BCI applications considers users' real-time operation with the external devices such as prosthetics, both-sided interactions and the actual experiences of the users as top priority [16]. The possibilities and boundedness of both invasive and non-invasive $\mathrm{BCI}$ platforms are the key factors that decide whether a BCI system is successful and its level of natural interaction with outer environment.

Currently the most successful BCI system in popularity is perhaps the artificial cochlear, which is a single-direction noninvasive platform that surpass the damaged hair cells and directly exert electric stimulation to auditory system and processed by the brain. Despite the high-end devices used in this technology, it stills only carries out simple function and doesn't allow feedback from the users' end. Their success is the integration of factors such as convenience, simple operation and low risk of infection from external devices. In fact, noninvasive methods such as EEG modes are not usually used alone as brain end signal sources, but are used in combination, or even cooperate with some non-EEG signals, such as muscle electrical signals (EMG) and eye electrical signals (EOG). Currently such noninvasive or hybrid mode BCI system are used in the following domains: replacement of lost motor function (e.g. hand prostheses), neural modulation or brain function modulation and speller. BCI could built an external signal pathways that replace the original pathway that assist the aiming movement of limbs with integration of electodes (often an electrodes hat). However, signal collection with only ERD or ERS (most common EEG methods) cannot guarantee high resolution and usually introduce noises, which make it hard to identify accurate movements. The problem could be alleviated through the combination of multiple methods like P300 and SSEP that together output a clearer command and path to external motors, patients also requires training to better operate the device. At present, the most widely used field of EEG is neuroscience, especially neurofeedback training and other neurological function treatment or regulation. Neural (EEG) biofeedback therapy is to collect EEG activity rhythms in various areas of the cerebral cortex, analyze the user's nerve status through EEG, and use visual and auditory signals to provide reward. It has been the treatment of for example ADHD and neural disorder, its also the potential solution to epilepsy, autism and etc. Patients who lost the capacity of communicating with outer environment could interact with the assistance of $\mathrm{BCI}$ systems, most commonly the speller based on P300 wave. The visual stimulus that the user pays attention to and the EEG induced by it are strictly positively correlated with a fixed time delay (300ms). The most famous example of BCI Speller is the speech assistance system used by Stephen Hawking (developed by Intel and swiftkey) [17]. EEG-based BCIs are also used or could potentially contribute to other fields such as entertainment and potential mental state monitoring or identifying.

By comparison, invasive brain-computer interfaces have the privileges of precise control, necessary user acceptance, and restoration of body surface sensation: prostheses (such as prostheses, eyes or teeth) will be accurately controlled by the output signals of the highlatitude brain-computer interfaces [18]. Additionally, common BCI systems only provides visual or auditory informational feedback, while the shortage of touch and proprioception often disrupt the normal functioning of the brain [19]. Through invasive systems, input information from the user end such as texture of the target could be directly delivered to the cortical sensory area. The information from the prosthesis may still not stimulate natural feelings, but the brain will learn to use these artificial information input channels. Due to the fact that the active potentials and local field potentials collected through invasive systems are generally better than EEG signals , many of the developing applications on prosthetics such as artificial or bionic limbs are based on invasive BCI systems that interfacing the neuromuscular system through nerve transfers and muscle interfacing [20]. 


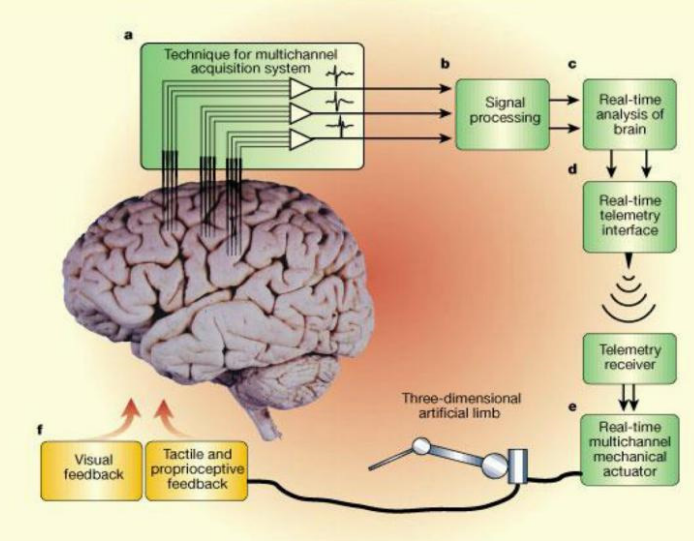

Fig. 3. Atificial limb system based on invasive BCIs

Signal transduction pathways are promised in both ways with such systems: central systems made up of brain and specific sections spinal cord transfers signals down the pathwaytp the peripheral systems through motor neuons, which could be capture by for example the Cuff electrodes, through targeted muscle reinnervation, the signals are converted to action potential which operate muscle cells; simultaneously, sensory information from the prosthetic end surpasses the amputated muscles, information that includes tactile perception, proprioception, pain, temperature and etc. are directly passed to spinal cords and could be further analysed by the brain.

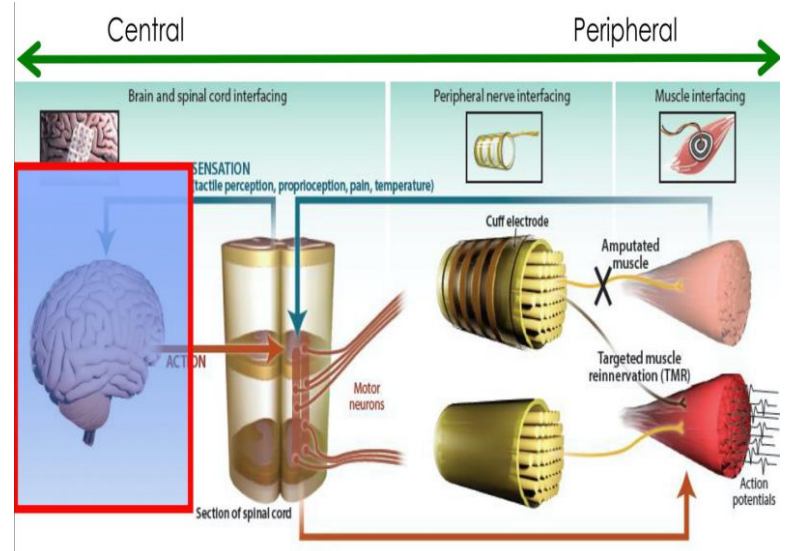

Fig. 4. Interfacing the neuromuscular sytem

The user acceptance of invasive brain-computer interfaces is generally lower than non-invasive braincomputer interfaces [21]. Invasive brain-computer interfaces will continue to be used for patients, while the current commercial brain-computer interfaces are all noninvasive. Although more research has proven that the risks of brain implantation as well as clinical issues has been overestimated, to obtain trust from publicity still requires improvement on information transmission rate, bio compatibility and long-term signal stability for invasive systems.

\section{Forecast of $\mathrm{BCls}$}

\subsection{Noninvasive Methods}

The dominant difficulty noninvasive systems facing is to amplify signals strength and improve their quality among loud noises. The electric field generated by neurons decays exponentially with distance, hence The activity of small neuron clusters cannot be detected or recorded by EEG with lower signal-to-noise ratio, which inevitably limits the usage of the systems. Except in the exceptional case of neuron bursts with action potentials, non-invasive signals are mainly used to analyze low-frequency neural activity $(<\approx 90 \mathrm{~Hz})$ [23]. Noises and spatial distortion further weaken the signals that could be detected by the external electodes. The limitations are specific to noninvasive or EEG based BCI systems and practically and theoretically could not be resolved. However, improvements on many aspects of noninvasive systems could still make it comparable with the invasive ones. The quality of the EEG signal (signal to noise ratio) mainly depends on the sensor (electrode), hardware circuit system (amplifier, processing chip and overall design, etc.). The privilege of noninvasive systems that it could cover the entire cerebral motor cortex could be further amplified through the reformation of detection methods, the combination of different noninvasive systems could also redempt the flaw of simple LFP signals. Data analysis and process is a dominant field that requires innovation. The week signals among noises and mutated through spatial distortion could be potential amplified or restored through multiple methods such as combining complex head models and algorithms with high-density EEG images to partially offset the distortion of the spatial signal and reduce the distortion to a certain noise level; applying source separation methods such as blind source separation could allow for accurate, real-time identification of motor neuron spikes in noninvasive or minimally invasive way.

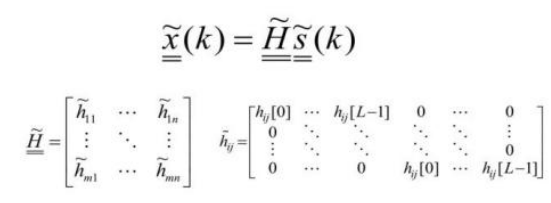

$\tilde{x}_{j}(k)=\left[x_{j}(k) x_{j}(k-1), \ldots, x_{j}(k-R)\right] \quad \tilde{s}_{i}(k)=\left[s_{i}(k), s_{i}(k-1), \ldots, s_{i}(k-(L+R-1))\right]$

Although upgrade the requirements for real-time analysis of large amounts of data have become higher for the hardware system, artificial intelligence (AI) based on machinery learning, such as support vector machines (SVM), can also greatly improve the accuracy of signal analysis and classification when handling the complex EEG signals with low spatial resolution. On the users' end, developments on ergonomics could significantly improve user experiences and further improve satisfication level. At present, the EEG-based BCI system is mainly composed of electrode caps (wet electrodes coated with conductive paste) as collection equipment, computers as processor, and multi-wire connection. Developing a comfortable external device using dry electrodes and being portable and sensitive to operate 
could eventually magnify the advantage of convenience of noninvasive systems and achieve widespread commercial application in more domains prior to invasive methods.

\subsection{Invasive Methods}

The dominant difficulties that invasive systems need to overcome rooted from our limitation of understanding towards neuroscience and the "neuron code". Most animal experiments are carried out on mice or primates. These experiments are only simple alternative primary reactions, and the corresponding functions of different neural parts (such as the pre-exercise cortex) have not been fully understood, which limits the actual function of the application. Once the relationships between as detailed as each motor neuron and motions has been discovered, users maybe obtain full control to the external devices control by such signals and the application won't be limited only to patients. Technique improvements are also not limited to implant smaller high-precision devices into the brain; transmit data through wireless signals which is the next target by NeuraLink according to Max Hodak, the president of NeuraLink; to charge implants external to the brain with wire connections or wireless, or choose the potential alternative method let implants utilize the energy from the body itself [24]. The BCI needs to adapt to the unstable signal environment and be equipped with a prosthesis with a better sensory-motor system. For implants implanted into the cortex, the main challenges are bio-compatibility, degradation of the implant over time, and failure of the implant due to damage to body tissues (such as rupture of the blood-brain barrier, neurodegenerative diseases, etc.) that are all potential threats that prevents chronic and stable data reception at a fixed location. Over years of working, although LFPs will also deteriorate, they still show better stability relative to APs which are mostly no longer recorded [25]. In order to improve lifespan and profitability, electrodes need to be designed in smaller sizes and resist immunity reaction with such as coated chemical factors that can integrate with neurons or apply flexible, bio-compatible materials to minimize stimulation to tissues [26]. Although the large-scale application of invasive BCIon humans haven't achieved, NeuraLink has the plan to have the permission from FDA for clinical trials which use robots to implant the entire data collection and processing system inside the skull of a paralysed patient that allws his to control mobile phones and computers. Once the above concerns be alleviated or solved through technology improvements, invasive BCIs could be perfectly embedded in human body and exchange information efficiently. When such implantation are proved to be safe over time with good bio-capacity, they may be accepted by most and become popular in a lot other domains other than medical fields such as entertainment and communication. But since it gives access to the brain, invasive $\mathrm{BCI}$ also needs to be responsible for potential moral issues such as privacy, hacker attract, remote control, and brain enhancement and social injustice, but hopefully these problems will not appear until such systems are advanced enough for commercial uses.

\section{Conclusion}

By comparing the two types of BCI platforms, a verdict could be made that noninvasive systems dominant the current BCI market because of its privilege of convenience and security, but in the long term, invasive systems that are premature in labs and surgical applications have the potential to overtake the dominant place in the commercial market due to its advantage of chronic data transferring and precision. Once the challenges of technology, social ethics, and neuroscience on invasive $\mathrm{BCIs}$ could be resolved with scientific development, users' worries may be relieved. At that time, the application of invasive BCIs will not be limited on the external environment and the users' status and will permeate in more application areas rather than solely medical fields.

\section{References}

1. J.R. Wolpaw, Encyclopedia of Neuroscience (2009) pp.429-437

2. C. Guger, Recent Advances in Brain-Computer Interface Systems (2011)

3. E. Musk. Journal of medical Internet research 21, 10 (2019)

4. H. Zhang, H. Wang, Computer Engineering and Applications 44, 24 (2008) pp. 228-230.

5. M. Rosa, T. Holder, Research using sheep leads to new device to record and stimulate the brain(2016), retrieved www.speakingofresearch.com

6. E. Niedermeyer, F.L. Da Silva, Electroencephalography: Basic Principles, Clinical Applications, and Related Fields. (Lippincott Williams \& Wilkins, 2004)

7. J. Wang, G. Xu, J. Xie, F. Zhang,L. Li, C. Han ,Y. Li,J. Brain Science Advances 4, 2 (2018) pp.156-167.

8. G. Muller-Putz, R. Leeb, M. Tangermann, J. Hohne, A, et al., Proceedings of the IEEE 103,6 (2015) pp.926-943.

9. G. Liu, W. Sun . Chinese Journal of Clinicians (2011).

10. T. Hinterberger, S. Schmidt, N Neumann, et al., Biomedical Engineering 51, 6 (2004) pp.1011-1018.

11. G. Bin, X. Gao, Y. Wang, Y. Li, B. Hong, S. Gao et al., Journal of neural engineering 8, 2 (2011)

12. E. Dong, G. Guo, C. Chen, Computer Engineering and Applications 53, 3 (2017) pp.154-159.

13. M. Middendorf, G. Mcmillan, G. Calhoum, et al., Rehabilitation Engineering 8, 2 (2000) pp.211-214.

14. J. Xie, G. Xu, J. Wang, et al., Plos one 7, 6 (2012)

15. H.J. Hwang, J.H. Lim, Y.J. Jung, et al., Development of an SSVEP-based BCI spelling system adopting a 
QWERTY-style LED keyboard. Journal of neuroscience methods 208, 1 (2012) pp.59-65.

16. G. Müller-Putz, R. Leeb, M. Tangermann, et al., Proceedings of the IEEE 103, 6 (2015) pp.926-943.

17. IBrain to allow Stephen Hawking to communicate through brainwaves alone (2012), retrieved www.newatlas.com

18. B. Wodlinger, J.E. Downey, E C. Tyler-Kabara, et al., Journal of neural engineering 12, 1 (2014)

19. F. Galán, S.N Baker, Frontiers in behavioral neuroscience 9 (2015)

20. S. Waldert, T. Pistohl, C. Braun, et al., Journal of Physiology-Paris 103, 3-5 (2009) pp.244-254.

21. C.H. Blabe, V. Gilja, C.A Chestek, et al., Journal of neural engineering 12,4 (2015)

22. L. Zrinzo, T. Foltynie, P. Limousin, et al., Journal of neurosurgery 116, 1 (2012) pp.84-94.

23. G. Waterstraat, M. Burghoff, T. Fedele, V. Nikulin, V., et al., Neuroimage 105 (2015) pp.13-20.

24. M.A. Hannan, S. Mutashar S, S.A Samad, et al., Biomedical engineering online 13, 1 (2014) pp.1-23.

25. A. Zhang, S. Malik, W.Q. Homer, M.L. Cash, et al. Journal of neural engineering 11, 4 (2014)

26. B.Gunasekera, T. Saxena, R. Bellamkonda, et al., ACS chemical neuroscience 6, 1 (2015),pp.68-83.

27. E. Musk, Neuralink. An integrated brain-machine interface platform with thousands of channels. bioRxiv. (2019) https://doi.org/10.1101/703801

28. S. Durai, P.T. Vanathi. EEG Signal Separation using Improved EEMD - Fast IVA Algorithm, Asian Journal of Research in Social Sciences and Humanities. 7(3):1230. (2017) DOI: 10.5958/22497315.2017.00239.8) 